\title{
PM versus IM Ti-5Al-5V-5Mo-3Cr Alloy in Mechanical Properties and Fracture Behaviour
}

\author{
Qinyang Zhao ${ }^{a}$, Fei Yang ${ }^{a *}{ }^{\oplus}$, Rob Torrens ${ }^{a}$, Leandro Bolzoni ${ }^{a}$ \\ ${ }^{a}$ Waikato Centre for Advanced Materials, School of Engineering, University of Waikato, Hamilton 3240, \\ New Zealand
}

Received: November 19, 2018; Revised: February 05, 2019; Accepted: April 29, 2019

\begin{abstract}
The comparisons of mechanical properties and fracture behaviour between as-consolidated PM Ti5553 alloy and as-cast IM Ti-5553 alloy were investigated through tensile, fracture toughness and impact toughness tests in this research. The slightly higher strength but much higher ductility and toughness can be identified in IM alloy specimens, which is also confirmed by the fracture behaviour of the specimens after mechanical tests. IM alloy specimens always exhibit the ductile dimple fracture mechanism in the different tests, while the fracture mechanism of PM alloy specimens indicates a high loading rate sensitivity, changing from the mixed ductile-brittle quasi-cleavage fracture into the brittle cleavage fracture mechanism accompanied by the remarkable decrease of impact toughness. The relatively low mechanical properties, especially the ductility and the brittle fracture behaviour of as-consolidated PM Ti-5553 alloy, are mainly explained by the differences in the initial microstructures between these two alloys.
\end{abstract}

Keywords: Powder metallurgy, Ti-5553 alloy, Mechanical property, Fracture behaviour.

\section{Introduction}

Titanium and its alloys are very promising materials in high-performance engineering applications due to their excellent properties, such as high specific strength, excellent corrosion resistance and biocompatibility ${ }^{1-3}$. The alloy Ti-5553 (Ti-5Al-5V-5Mo-3Cr) displays an ultra-high strength, better hardenability and larger processing windows comparing to other metastable $\beta$ titanium alloys, and it has been attracted with much attention in the field of engineering applications ${ }^{4-6}$. However, the relatively high overall cost, from raw materials to machining, limits their widespread uses ${ }^{7,8}$. Powder metallurgy (PM) approaches are regarded as cost-effective processing techniques for producing titanium products, which not only have a possibility to reduce the product cost, also have some extra advantages like the freedom in selection of product's composition ${ }^{9}$.

The mechanical properties and the fracture behaviour are vital aspects in the structural applications of titanium alloys as they can influence the failure and lifetime of the materials, and they become primary concerns in the development and the feasibility of PM titanium approaches. Although the mechanical properties of the Ti-based parts prepared through HIP (hot isostatic pressing) ${ }^{10}$, PS (pressing-sintering) ${ }^{11}, \mathrm{BE}$ (blended elemental) ${ }^{12}$ and SLM (selective laser melting) ${ }^{13}$ routes are examined to meet the ASTM standard, It was reported that PM parts are still suffering from some shortages in mechanical properties compared with ingot metallurgy (IM) products including low ductility, low fatigue resistance and insufficient fracture toughness ${ }^{14}$.

*e-mail: fei.yang@waikato.ac.nz
There were significant efforts to study the mechanical properties and fracture behaviour of IM metastable $\beta$ titanium alloys including Ti-5553 alloys during tensile, fracture toughness and impact tests. Ghosh et al. ${ }^{15}$ investigated the influence of microstructure on fracture toughness of Ti-5553 alloy and pointed out that the presence of $\alpha$ phase can improve fracture toughness significantly. Shekhar et al. ${ }^{16}$ performed different heat treatment regimens on Ti-5553 alloy and examined the effect of the microstructure on the fractography of tensile tests. The influences of the isothermal thermal-mechanical processing strain on the fracture behaviour during impact toughness test of Ti-5Al-2Sn-2Zr-4Mo-4Cr $(\alpha+\beta)$ titanium alloy were investigated by Xu et al ${ }^{17}$. In terms of PM titanium alloy, Yang et al. ${ }^{18}$ prepared PM metastable $\beta$ Ti-10V-2Fe-3Al titanium alloy using two kinds of master alloy powders and investigated the differences in the tensile fracture behaviour. Zheng et al. ${ }^{19}$ manufactured pure titanium rods through rapid powder compact extrusion approach and found that the extrusion temperature has a considerable effect on the tensile properties and tensile fractography. The relationship between the fracture behaviour and residual porosity of powder metallurgy (Pressing-Sintering) Ti-6Al-7Nb biomedical titanium alloy obtained by different sintering temperature was explored by Bolzoni et al ${ }^{20}$.

In these related works, the mechanical properties and fracture behaviour of IM and PM titanium alloys were studied and discussed, but there is no work compared the mechanical properties directly between PM and IM alloys comprehensively, nor the comparisons of fracture behaviour were referred. Moreover, the published literature which studied the fracture behaviour of PM titanium alloys were mainly focused on PM pure titanium and Ti-6Al-4V alloy, but PM metastable $\beta$ titanium alloys were rarely involved. 
Therefore, the comparisons of mechanical properties and fracture behaviour of PM and IM titanium alloys especially metastable $\beta$ titanium alloys are necessary to perform for the titanium industry to identify the differences and understand underlying fracture mechanisms of PM and IM titanium alloy in mechanical testing with the same ideal chemical composition.

A rapid thermomechanical route was utilized in this paper to produce as-consolidated PM Ti-5553 alloy billets with blended powder mixtures containing HDH-Ti powder and master alloy powders. The mechanical tests at room temperature including tensile, fracture toughness and impact toughness testing were conducted to compare the mechanical properties and fracture behaviour with as-cast IM Ti-5553 alloy manufactured by traditional VAR melting and casting. The microstructure, mechanical properties, fractographic characterization and fracture mechanisms of PM and IM Ti-5553 alloys are investigated and discussed.

\section{Experimental}

The starting materials for synthesising PM Ti-5553 (Ti5Al-5V-5Mo-3Cr) alloy billets were hydride-dehydrided (HDH) titanium powder (-250mesh, purity: 99.6\%), Al powder (purity: 99.9\%), Al35-V65, Al15-Mo85 and Al30Cr70 (wt\%) master alloy powders $(75 \mu \mathrm{m}$, commercial purity) supplied by Dalian Rongde Company, PR China. The powder mixture with the target composition was first mixed in a V-shape blender at a speed of $60 \mathrm{rpm}$ for 90 mins, then was compacted into a cylindrical shape, with a dimension of $56 \mathrm{~mm}$ in diameter and $52 \mathrm{~mm}$ in height, by an uniaxial mechanical press under $400 \mathrm{MPa}$, at $250{ }^{\circ} \mathrm{C}$ in air. After that, the powder compact was heated up to 1250 ${ }^{\circ} \mathrm{C}-1300{ }^{\circ} \mathrm{C}$ in argon atmosphere chamber, with controlled oxygen content below $200 \mathrm{ppm}$, using induction furnace, held at the temperature for 10 mins and then hot pressed using 100 ton hydraulic press under a pressure of about 400 MPa, followed by flow-argon cooling to room temperature. The relative density for the Ti-5553 powder compact and hot-pressed billet was about $84 \%$ and $98 \%$, respectively. The IM Ti-5553 alloy ingot $(160 \mathrm{~mm}$ in diameter and $35 \mathrm{~kg}$ in weight), was produced by vacuum arc remelting (VAR) process and casting. The chemical compositions (measured by the method of inductively coupled plasma atomic emission spectrometry) of as-consolidated PM and as-cast IM Ti-5553 alloy are listed in Table 1.

Table 1. Chemical compositions of as-consolidated PM and ascast IM Ti-5553 (wt\%).

\begin{tabular}{ccccccc}
\hline & $\mathrm{Ti}$ & $\mathrm{Mo}$ & $\mathrm{Al}$ & $\mathrm{Cr}$ & $\mathrm{V}$ & $\mathrm{O}$ \\
\hline $\mathrm{PM}$ & Bal. & 4.94 & 4.99 & 2.90 & 4.93 & 0.36 \\
$\mathrm{IM}$ & Bal. & 5.02 & 5.14 & 3.10 & 5.03 & 0.08 \\
\hline
\end{tabular}

Tensile tests were conducted at room temperature using an Instron-5982 universal testing machine, and the $4 \mathrm{~mm} \times 2.5$ $\mathrm{mm}$ rectangle cross-section tensile specimens were cut from both PM and IM Ti-5553 alloy billets and had a gauge length of $15 \mathrm{~mm}$. The strain was measured using an extensometer, and the strain rate of the tensile testing was $1 \times 10^{-3} \mathrm{~s}^{-1}$.

The single notched specimens were wire cut from the alloys followed by machining and polishing to perform fracture toughness tests on an Instron-5982 electronic universal test machine at room temperature. The specimen had the thickness $\mathrm{B}=2.5 \mathrm{~mm}$, width $\mathrm{W}=4.5 \mathrm{~mm}$, loading span $1=16 \mathrm{~mm}$, and the notch depth $\mathrm{a}=2.0 \mathrm{~mm}$ which provides plane-strain conditions at the notch tip, and the tests were conducted with a loading rate of $0.5 \mathrm{~mm} / \mathrm{min}$. The load-displacement curves were recorded during the test, and the apparent fracture toughness $\mathrm{K}_{\mathrm{Q}}$ can be calculated using formula (1) and (2). However, $\mathrm{K}_{\mathrm{Q}}$ doesn't necessarily equal $\mathrm{K}_{\mathrm{IC}}$ only if the small-scale yielding and plane strain conditions at the crack tip of the test specimen are met, while in this work the values of $\mathrm{K}_{\mathrm{Q}}$ were utilized to compare the fracture toughness of PM and IM alloys.

$$
\begin{gathered}
\mathrm{K}_{\mathrm{Q}}=\left(\mathrm{F}_{\mathrm{Q}} / B W^{1 / 2}\right) \times \mathrm{f}(\mathrm{a} / \mathrm{W}) \\
\mathrm{f}(\mathrm{a} / \mathrm{W})=(2+\mathrm{a} / \mathrm{W}) \times \\
\underline{0.866+4.64(a / W)-13.32(a / W)^{2}-14.72(a / W)^{3}-5.6(a / W)^{4}}
\end{gathered}
$$

Charpy u-notch impact toughness specimens $(50 \mathrm{~mm}$ length, square-shaped cross section with $10 \mathrm{~mm}$ side length, $2 \mathrm{~mm}$ depth $45^{\circ} \mathrm{V}$-shape notch) were prepared from both $\mathrm{PM}$ and IM Ti-5553 alloys and the testing was performed at room temperature using a NJ780C pendulum bob impacttesting machine with a maximum energy rating of $400 \mathrm{~J}$, and the impact velocity is $5.0 \mathrm{~m} / \mathrm{s}$.

Optical microscopy (OM, Olympus PMG3) and scanning electron microscopy (SEM, JSM-6460) were used to examine the microstructures and fracture surfaces of the PM and IM Ti-5553 alloys specimens after mechanical tests. The ground and polished metallographic surfaces of the samples were etched in a modified Kroll's reagent consisting of $2 \mathrm{vol} \%$ $\mathrm{HF}, 4 \mathrm{vol} \% \mathrm{HNO}_{3}, 94 \mathrm{vol} \% \mathrm{H}_{2} \mathrm{O}$.

\section{Results and Discussion}

\subsection{The initial microstructure and mechanical properties of PM and IM Ti-5553 alloys}

Fig. 1 shows the initial microstructures of the as-consolidated PM and as-cast Ti-5553 alloys, it can be seen that the PM Ti5553 alloy is consisted of primary equiaxed $\beta$ phase, with an average grain size of about $100 \mu \mathrm{m}$, and a small amount of precipitation phases can be observed in the $\beta$ matrix (Fig. 1a). 
A similar microstructure for the IM Ti-5553 alloy can be observed in Fig. 1b, with the primary differences of a larger amount of precipitates inside $\beta$ grains and coarser equiaxed $\beta$ phase (about $1000 \mu \mathrm{m}$ ) than those of the PM Ti-5553 alloy.

The results of tensile, fracture toughness and impact toughness tests for the as-consolidated PM and as-cast IM Ti-5553 alloys are the listed in Table 2. It is clear that the IM alloy has overall better mechanical properties than the PM alloy, in particular the ductility and toughness, with a value of $3.8 \%$ for the ductility, and $64 \mathrm{MPa} \cdot \sqrt{\mathrm{m}}$ and $19 \mathrm{~J} / \mathrm{cm}^{3}$ for the fracture and impact toughness, respectively. However, the PM counterpart has a ductility of $2.1 \%$, and fracture and impact toughness of $28 \mathrm{MPa} \cdot \sqrt{\mathrm{m}}$ and $4 \mathrm{~J} / \mathrm{cm}^{3}$, respectively.

\subsection{Fracture behaviour of tensile tests}

The fracture surfaces of tensile specimens of the IM and PM Ti-5553 alloys are exhibited in Fig. 2. A relative flat fracture surface composed by continuous small fracture facets and tear ridges can be observed in the macroscopical images of PM alloy (Fig. 2a), the detailed fracture surface morphologies can be revealed in Fig. $2 b$ and 2c, typical cleavage fracture facets with river-like patterns divided by tear ridges, some ununiformly distributed shallow and small dimples can be clearly identified. These fracture surface morphologies indicate that the fracture mechanism of PM alloy during the tensile tests is quasi-cleavage mixed ductile-brittle fracture.

However, IM alloy specimen displays a significant tortuous fracture surface after tensile tests in macroscopical images shown in Fig. 2d, and the homogenous deep dimples spread over fluctuant fracture surface can be observed in enlarged images in Fig. 2e and 2f. Entire ductile fracture mechanism with a large plastic deformation and energy absorption can be speculated from the fracture surface features of IM alloy.

The macroscopical and microscopical fracture surfaces morphology and the defined dominated fracture mechanisms suggest the higher tensile properties of IM Ti-5553 alloy than PM Ti-5553 alloy especially in ductility, which agrees with the obtained tensile properties Table 2 .

\subsection{Fracture behaviour of fracture toughness tests}

Fig. 3 presents the fracture surfaces of the specimens of the PM and IM Ti-5553 alloys for fracture toughness test at room temperature. First of all, similar to the tensile fracture surface in macroscopical scale, flat and even cracking surface can be seen in PM alloy fracture toughness specimen in Fig. 3a. Large cleavage facets conjunction with tear ridges and secondary cracks can be found in Fig. $3 \mathrm{~b}$ and $3 \mathrm{c}$, while Fig. $3 \mathrm{c}$ also presents a number of small and irregular dimples arranged between flat cleavage facets. A lower energy consummation of $\mathrm{PM}$ alloy during the crack propagation and the cleavage dominated cracking mechanism can be inferred and identified during the fracture toughness test of PM alloy.

As for IM alloy, as shown in Fig. 3d, topography morphology of the rough cracking propagation surface is clear in the macroscopical scale fracture surface images, suggesting a higher energy consumption during the cracking propagation than PM alloy. Meanwhile, the regular and uniform distributed dimples in the segment enlarged fracture surface in Fig. 3e and $3 \mathrm{f}$ demonstrate the distinct ductile cracking mechanism of IM alloy.

Table 2. Mechanical properties of as-consolidated PM and as-cast IM Ti-5553 alloy.

\begin{tabular}{|c|c|c|c|c|c|}
\hline \multirow{2}{*}{ Alloy Conditions } & \multicolumn{3}{|c|}{ Tensile properties } & \multirow{2}{*}{$\mathrm{K}_{\mathrm{Q}}(\mathrm{MPa} \cdot \sqrt{ } \mathrm{m})$} & \multirow{2}{*}{ Impact toughness $\left(\mathrm{J} / \mathrm{cm}^{2}\right)$} \\
\hline & Yield stress (MPa) & Ultimate stress (MPa) & Elongation (\%) & & \\
\hline PM alloy & 935 & 1008 & 2.1 & 28 & 4 \\
\hline IM alloy & 1126 & 1220 & 3.8 & 64 & 19 \\
\hline
\end{tabular}
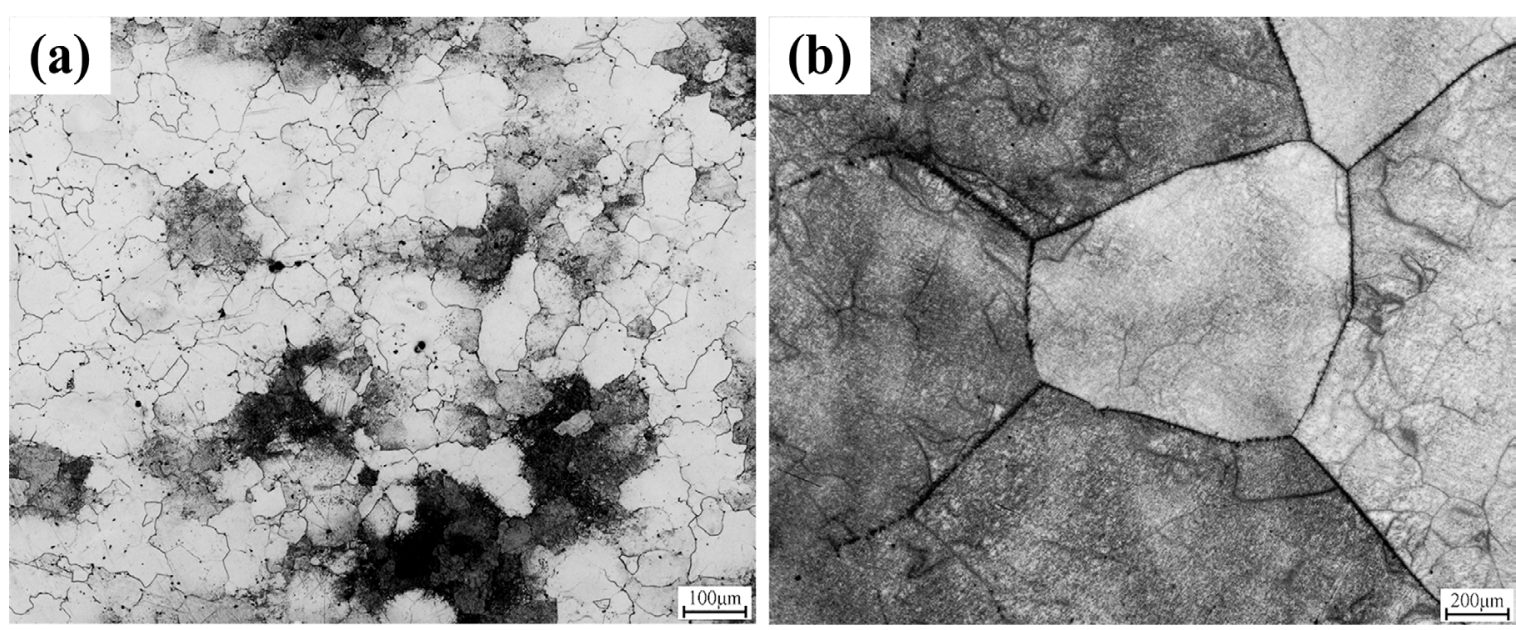

Figure 1. Initial microstructures of Ti-5553 alloys: (a) as-consolidated PM alloy; (b) as-cast IM alloy. 

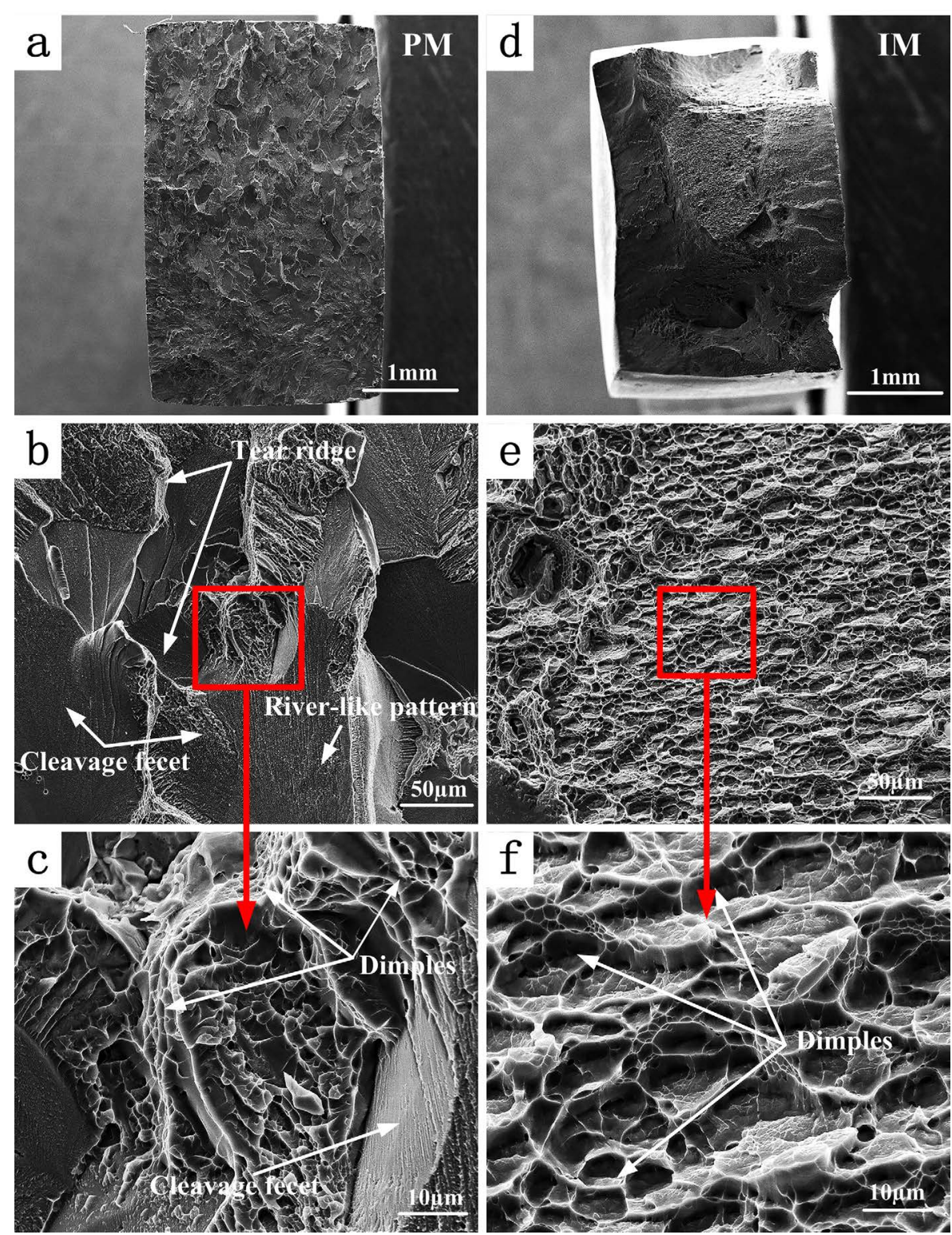

Figure 2. SEM images of macroscopical and microscopical fracture surface morphologies of the specimens after tensile tests of as-consolidated PM and as-cast IM Ti-5553 alloys: (a) PM macroscopical scale; (b) and (c) PM microscopical scale; (d) IM macroscopical scale; (e) and (f) IM microscopical scale.

By analysing the cracking surface morphology of the fracture toughness specimens, there is no doubt that PM alloy exhibits a much lower fracture toughness than IM alloy due to the ease of cracking propagation and brittle fracture mechanism. However, the appearance of the small dimples in the PM specimen suggest the crack propagation is inhibited by these dimples and increase the fracture toughness value to some extent ${ }^{21}$.

\subsection{Fracture behaviour of impact toughness tests}

The impact toughness specimens of IM and PM Ti-5553 alloy after the tests can firstly be given a macroscopical view to analyse the dynamic fracture behaviour of the alloys. From Fig. 4a and 4d, it is clear that dynamic fracture surfaces of these two different alloys show entirely different features. The IM specimen has a tortuous surface (Fig. 4b), demonstrating that a longer cracking path during the impact fracture process of the IM alloy with higher energy consumption and reflecting in the change of the amplitude of the crack path ${ }^{17}$ during the impact test. However, a relatively even and smooth impact fracture surface (Fig. 4a) can be observed in PM specimen which absorbs relatively lower impact energy during the test. 

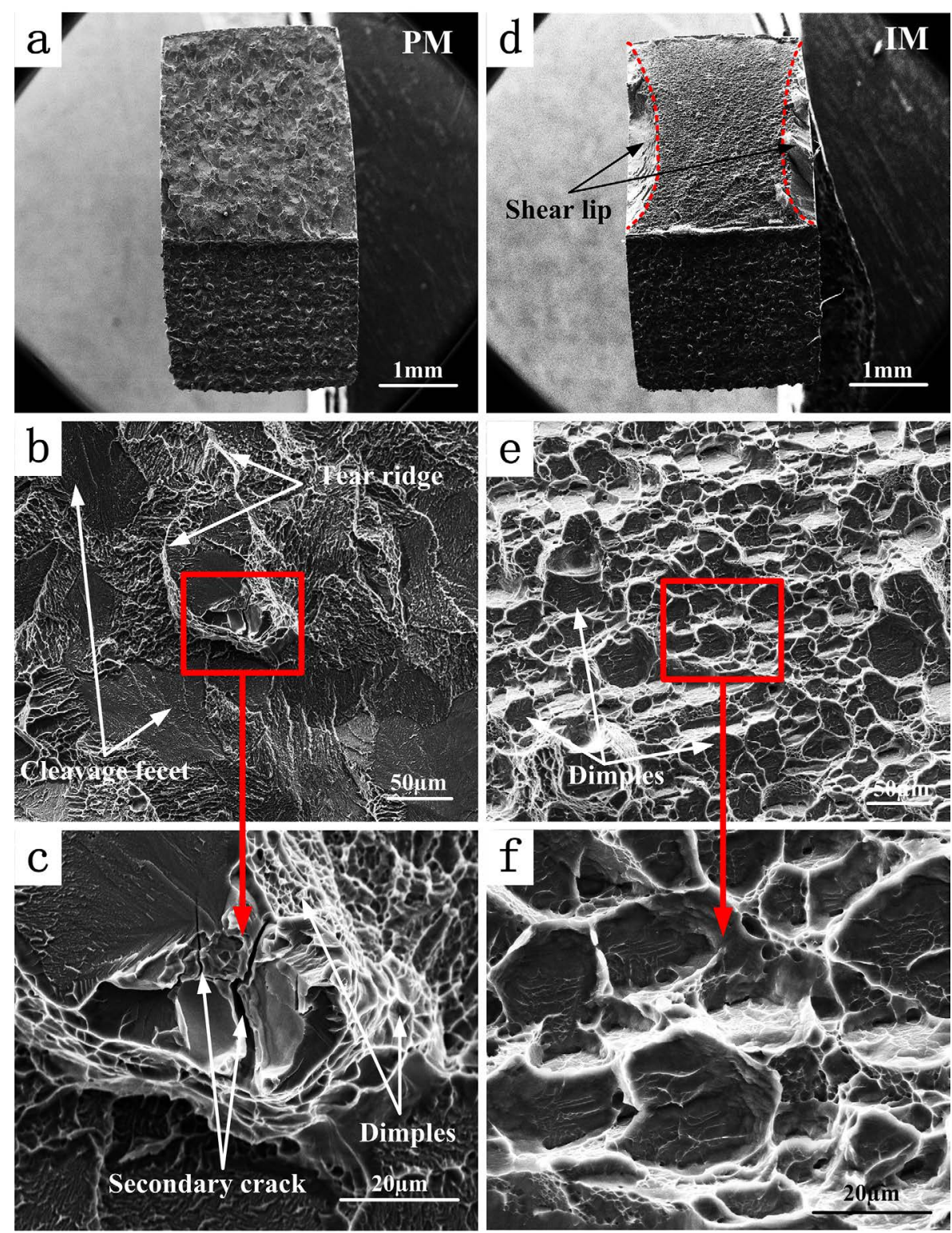

Figure 3. SEM images of macroscopical scale and microscopical scale fracture surface morphologies of the specimens after fracture toughness tests of as-consolidated PM and as-cast IM Ti-5553 alloys: (a) PM macroscopical scale; (b) and (c)PM microscopical scale; (d) IM macroscopical scale; (e) and (f) IM microscopical scale.

More detailed impact toughness fracture surface morphologies of PM and IM Ti-5553 alloys are shown in the segment enlarged surface images. As shown in Fig. $4 \mathrm{~b}$, big and uninterrupted cleavage facets accompanied by tear ridges distribute tightly in the impact fracture surface of PM alloy specimen. Meanwhile, it worth noticing that some areas are sticking out from the flat fracture surface accompanied by the secondary cracks surrounded by some tiny and shallow dimples in PM alloy impact fracture surface (Fig. 4c). These features suggest that the cleavage brittle intergranular fracture is the dominated mechanism in PM alloy during the impact toughness test. On the contrary, as shown in Fig. $4 \mathrm{e}$ and $4 \mathrm{f}$, deep and big dimples accompanied by several ravines are speared uniformly over the impact fracture surface which characterizes the ductile fracture mechanism and signifies the considerable improvement of the impact toughness toughness for IM alloy.

Corresponding to the mechanical properties in Table 2, the much higher impact toughness of IM alloy than PM alloy can be certified by the fracture behaviour and mechanisms in this section. 

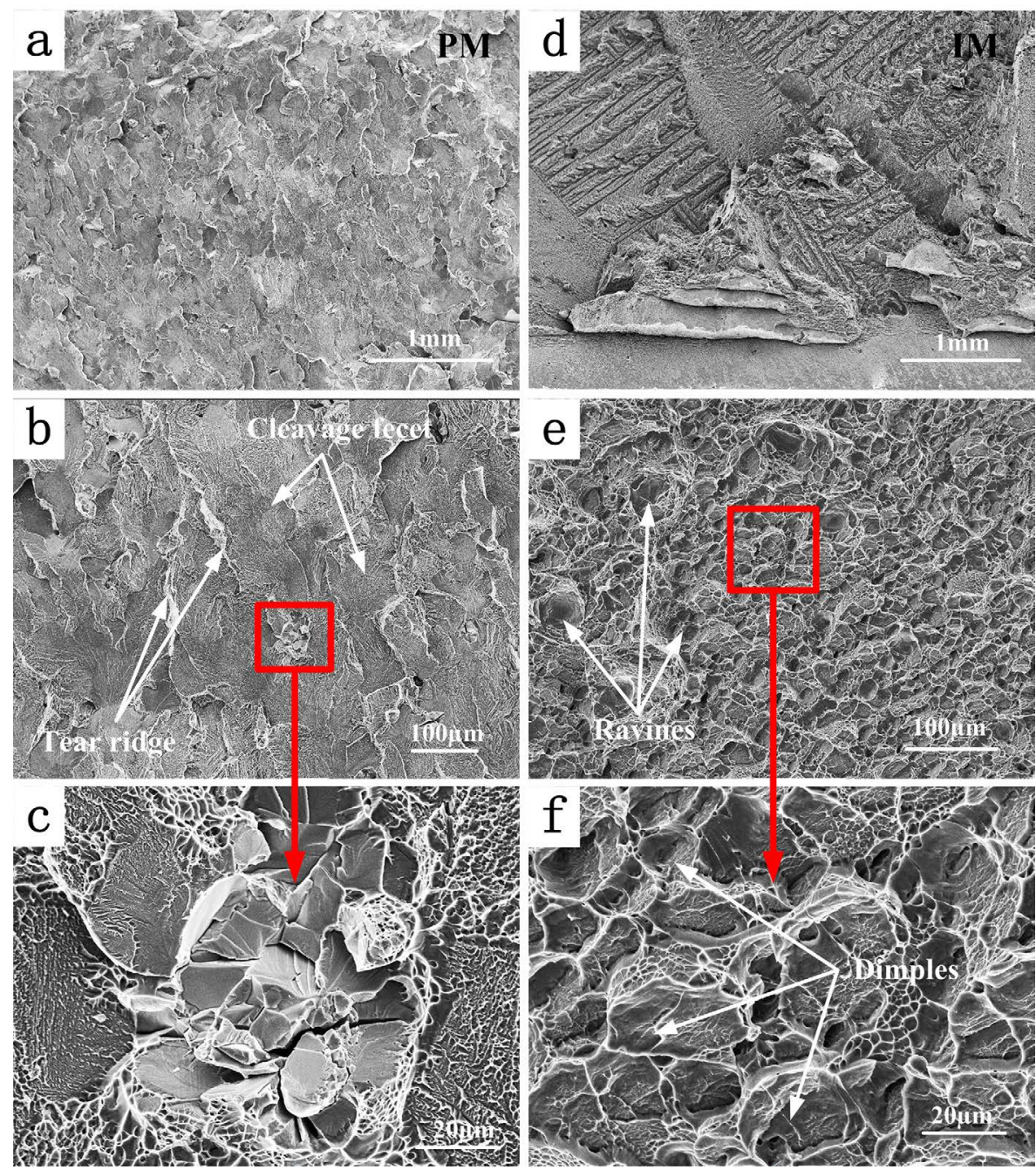

Figure 4. SEM images of macroscopical scale and microscopical scale fracture surface morphologies of the specimens after impact toughness tests of as-consolidated PM and as-cast IM Ti-5553 alloys: (a) PM macroscopical scale; (b) and (c)PM microscopical scale; (d) IM macroscopical scale; (e) and (f) IM microscopical scale.

\subsection{The analysis of the differences between PM and IM alloy}

By analysing and comparing the room temperature mechanical properties of as-cast IM and as-consolidated PM Ti-5553 alloys, the remarkable gaps between tensile ductility, fracture toughness and impact toughness can be clearly viewed. These gaps have been verified by the fractographic characterization using SEM, IM alloy specimens are mainly dominated by dimple ductile fracture mechanism, while quasi-cleavage and cleavage brittle fracture mechanisms are revealed in PM specimens, as shown in Figs. 2 4.

It worth noticing that, after the fracture toughness test, obvious shear lips can be identified in the macroscopical fracture surface of IM alloy in Fig. 3d, but they are not observed in PM specimen. It is well known that plane stress conditions can be realized at the notched side surface of fracture toughness specimen, and with the increasing distance from the surface, the stress triaxiality during the static loading in the fracture toughness tests goes up obviously. The areas with low-stress triaxiality are tended to suffer from shear cracking which involves a large amount of plastic deformation and provide sizable fracture resistance, the 'shear lips' is one of the features of the shear cracking in the macroscopical scale of the fracture toughness specimen fracture surface ${ }^{22}$. The appearance of the shear lips indicates a higher plastic deformation during the test and a higher fracture toughness of IM alloy than PM alloy.

The relatively low ductility and the cleavage dominated fracture mechanisms of PM Ti-5553 alloy can be mainly attributed to the initial microstructure and the residual porosity ${ }^{14}$. The glide of dislocations and the propagation of micro-cracks are easier to realize during the deformation of PM alloy than IM alloy as less $\alpha$ phase precipitates in the microstructure. The precipitation of $\alpha$ phase in $\beta$ matrix can 
offer the obstruction effect for cracks and dislocation and thus improve the ductility and fracture resistance dramatically ${ }^{9,23}$.

As for the residual micropores, although the relative density of the as-consolidated PM Ti-5553 alloy in this work can reach $98 \%$, there are still some residual pores in the microstructure of alloy, the distribution and morphology of residual micropores in the alloy matrix are shown in Fig. 5. Circular and near-circular pores with the diameter from $2 \mu \mathrm{m}$ to $5 \mu \mathrm{m}$ appear both on the grain boundaries and inside the $\beta$ grain matrix. These residual pores may act as the defects and become the crack originals or provide the crack propagation path with low energy consumption, and thus reduce the tensile ductility, fracture toughness, impact toughness and strength of as-consolidated PM alloy ${ }^{24}$.
As-consolidated PM Ti-5553 alloy also exhibits not exactly the same fracture mechanism during the static and dynamic mechanical tests. Mixed ductile-brittle and quasi-cleavage transgranular fracture characteristics are identified in tensile and fracture toughness tests specimens, while complete brittle cleavage fracture mechanism with the appearance of intergranular features is obvious in the impact toughness test specimen, which indicates that the ductility of PM alloy is reduced dramatically with the increase of loading rate. However, the absence of this kind of mechanism change in as-cast IM Ti-5553 alloy illustrates that the ductility and fracture mechanism of IM alloy are not as sensitive as PM alloy to the loading rate.
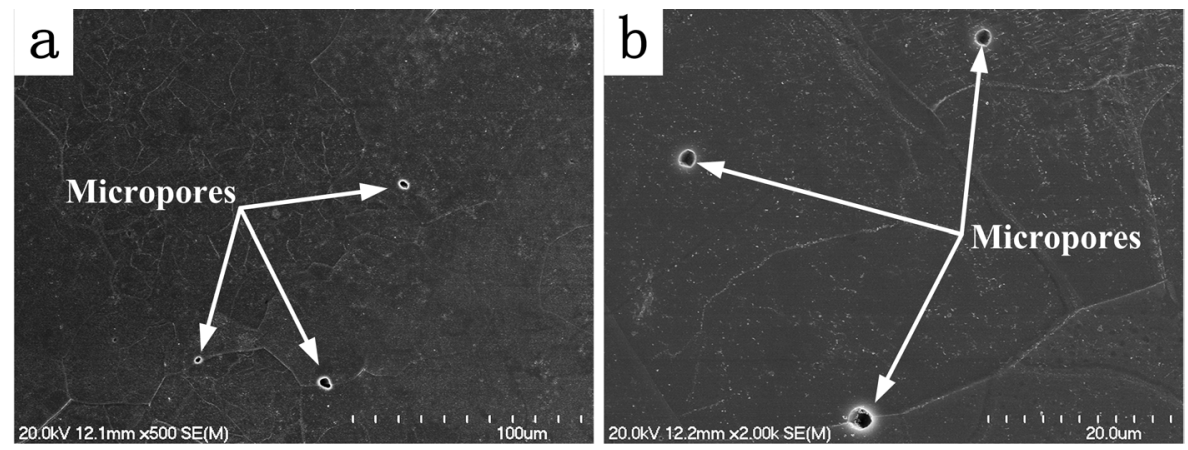

Figure 5. SEM images of the residual micropores in as-consolidated PM Ti-5553 alloy.

\section{Conclusions}

The comparisons of the room temperature mechanical properties and fracture behaviour between as-consolidated PM Ti-5553 alloy and as-cast IM Ti-5553 alloy were carried out by the tensile, fracture toughness and impact toughness tests, and the following conclusions can be drawn:

(1) IM alloy performs slightly higher tensile strength including yield stress and ultimate stress than PM alloy. However, much higher ductility during the quasi-static tensile and fracture toughness tests can be obtained in IM alloy. The ductility gap between IM and PM alloy becomes more significant in the dynamic loading impact toughness test.

(2) All the PM alloy specimens after the mechanical tests suggest flatter fracture surfaces than IM alloy specimens in macroscopical views, indicates the relatively low ductility and toughness of PM alloy.

(3) The fracture mechanism of PM alloy during the tests is brittle cleavage dominated mechanism, while ductile dimple fracture characteristics can be observed in IM alloy specimens. The differences in the initial microstructure and the residual porosity are the main issues to blame for the lower ductility and cleavage fracture mechanism of PM alloy during the mechanical test.
(4) Unlike the consistent ductile dimple fracture mechanism of IM alloy at different loading rates, the fracture behaviour and mechanisms of PM alloy are sensitive to the loading rate. Mixed ductile-brittle and quasi-cleavage transgranular fracture mechanism change into complete brittle cleavage fracture mechanism with the appearance of intergranular features as as increasing the loading rate of PM alloy

\section{Acknowledgements}

The funding and support from New Zealand Ministry of Business, Innovation and Employment (MBIE, UOWX1402) are acknowledged gratefully.

\section{References}

1. Banerjee D, Williams JC. Perspectives on Titanium Science and Technology. Acta Materialia. 2013;61(3):844-879.

2. Froes FH. A historical perspective of titanium powder metallurgy. In: Qian M, Froes FH, eds. Titanium Powder Metallurgy. Oxford: Butterworth-Heinemann; 2015. p. 1-19.

3. Huang C, Zhao Y, Xin S, Zhou W, Li Q, Zeng W. Effect of microstructure on torsion properties of Ti-5Al-5Mo-5V-3Cr-1Zr alloy. Materials Science and Engineering: A 2017;682:202-210. 
4. Fanning JC. Properties of TIMETAL 555 (Ti-5Al-5Mo-5V-3Cr0.6Fe). Journal of Materials Engineering and Performance. 2005;14(6):788-791.

5. Nag S, Banerjee R, Srinivasan R, Hwang JY, Harper M, Fraser HL. $\omega$-Assisted nucleation and growth of a precipitates in the Ti-5Al-5Mo-5V-3Cr-0.5Fe $\beta$ titanium alloy. Acta Materialia. 2009;57(7):2136-2147.

6. Zhu Y, Zeng W, Zhao Y, Shu Y, Zhang X. Effect of processing parameters on hot deformation behavior and microstructural evolution during hot compression of Ti40 titanium alloy. Materials Science and Engineering: A. 2012;552:384-391.

7. Liu Y, Chen LF, Tang HP, Liu CT, Liu B, Huang BY. Design of powder metallurgy titanium alloys and composites. Materials Science and Engineering: A. 2006;418(1-2):25-35.

8. Froes FH, Friedrich H, Kiese J, Bergoint D. Titanium in the family automobile: The cost challenge. JOM. 2004;56(2):40-44.

9. Yang F, Gabbitas B, Dore M, Ogereau A, Raynova S, Bolzoni L. On microstructural evolution and mechanical properties of Ti-5Al-5V-5Mo-3Cr alloy synthesised from elemental powder mixtures. Materials Chemistry and Physics. 2018;211:406-413.

10. Xu L, Guo R, Bai C, Lei J, Yang R. Effect of Hot Isostatic Pressing Conditions and Cooling Rate on Microstructure and Properties of Ti-6Al-4V Alloy from Atomized Powder. Journal of Materials Science \& Technology. 2014;30(12):1289-1295.

11. Jia MT, Zhang DL, Gabbitas B, Liang JM, Kong C. A novel Ti-6Al-4V alloy microstructure with very high strength and good ductility. Scripta Materialia. 2015;107:10-13.

12. Froes FH. Powder metallurgy of titanium alloys. In: Chang I, Zhai Y, eds. Advances in Powder Metallurgy - Properties, Processing and Applications. Oxford: Woodhead Publishing; 2013. p. 202-240.

13. Vrancken B, Thijs L, Kruth JP, Van Humbeeck J. Microstructure and mechanical properties of a novel $\beta$ titanium metallic composite by selective laser melting. Acta Materialia. 2014;68:150-158.

14. Wang H, Fang ZZ, Sun P. A critical review of mechanical properties of powder metallurgy titanium. International Journal of Powder Metallurgy. 2010;46(5):45-57.
15. Ghosh A, Sivaprasad S, Bhattacharjee A, Kar SK. Microstructurefracture toughness correlation in an aircraft structural component alloy Ti-5Al-5V-5Mo-3Cr. Materials Science and Engineering: A. 2013;568:61-67.

16. Shekhar S, Sarkar R, Kar SK, Bhattacharjee A. Effect of solution treatment and aging on microstructure and tensile properties of high strength $\beta$ titanium alloy, Ti-5Al-5V-5Mo-3Cr. Materials \& Design. 2015;66(Pt B):596-610.

17. Xu JW, Zeng WD, Zhao YW, Jia ZQ. Effect of microstructure evolution of the lamellar alpha on impact toughness in a twophase titanium alloy. Materials Science and Engineering: A. 2016;676:434-440.

18. Yang YF, Luo SD, Schaffer GB, Qian M. Sintering of Ti-10V$2 \mathrm{Fe}-3 \mathrm{Al}$ and mechanical properties. Materials Science and Engineering: A. 2011;528(22-23):6719-6726.

19. Zheng YF, Yao X, Liang JM, Zhang DL. Microstructures and Tensile Mechanical Properties of Titanium Rods Made by Powder Compact Extrusion of a Titanium Hydride Powder. Metallurgical and Materials Transactions A. 2016;47(4):1842-1853.

20. Bolzoni L, Ruiz-Navas EM, Gordo E. Evaluation of the mechanical properties of powder metallurgy Ti-6Al-7 Nb alloy. Journal of the Mechanical Behaviour of Biomedical Materials. 2017;67:110-116.

21. Srinivasu G, Natraj Y, BhattacharjeeA, Nandy TK, Nageswara Rao GVS. Tensile and fracture toughness of high strength ß Titanium alloy, Ti-10V-2Fe-3Al, as a function of rolling and solution treatment temperatures. Materials \& Design. 2013;47:323-330.

22. Shi X, Zeng W, Zhao Q. The effect of surface oxidation behavior on the fracture toughness of Ti-5Al-5Mo-5V-1Cr-1Fe titanium alloy. Journal of Alloys and Compounds. 2015;647:740-749.

23. Semiatin SL, Bieler TR. The effect of alpha platelet thickness on plastic flow during hot working of TI-6Al-4V with a transformed microstructure. Acta Materialia. 2001;49(17):3565-3573.

24. Bourcier RJ, Koss DA, Smelser RE, Richmond O. The influence of porosity on the deformation and fracture of alloys. Acta Metallurgica. 1986;34(12):2443-2453. 